\title{
Sensor concepts for the detection of explosives with selective layers based on molecularly imprinted polymers
}

\author{
J. Hürttlen, G. Bunte, D. Röseling, M. Heil, H. Krause \\ Fraunhofer Institute for Chemical Technology ICT \\ Joseph-von-Fraunhofer-Str. 7, 76327 Pfinztal, Germany, \\ juergen.huerttlen@ict.fraunhofer.de
}

\begin{abstract}
:
In concerns with national security there are needs for inexpensive, rapid, high sensitive and selective sensors. In this respect, e.g. mass-sensitive devices (QCMs) coated with substance specific molecularly imprinted polymers (MIPs) look promising to achieve low-cost detection devices capable of detecting explosives vapours fast, sensitive and reliable. ICT works on the development of MIPs as specific sensing layers to detect common ingredients in improvised explosives like 2,4,6-TNT, 2,4DNT, EGDN, tagging substances like DMNB or the self-made explosive most frequently used by terrorists, TATP. Preliminary results for 2,4,6-TNT and 2,4-DNT and possible concepts for MIP-based low-cost sensors are presented.
\end{abstract}

Key words: Explosives detection, low cost sensor, molecular imprinted polymers, coating

\section{Introduction}

Recent terrorist attacks such as the bombing in New York at 11.9.2001, suicide bombings in the middle east or e.g. in London Underground transportation systems in July 2005 show that the today used detection techniques for explosives (Imaging by X-ray, neutron activation techniques, IMS, NQR,) are inadequate and have to be improved as well as new counter-action / detection concepts have to be developed. The main ingredients in improvised explosives like 2,4,6-TNT, 2,4-DNT, $\mathrm{NGI}$, EGDN, tagging substances like DMNB or the self-made explosive most frequently used by terrorists, TATP, exhibit vapour pressures in the $\mathrm{ppb}$ to percentage range which in principle enable a possible detection of these substances via vapour trace detection methods. Therefore there are needs for inexpensive, rapid, high sensitive and selective sensors. In this respect, our approach is to develop substance-specific sensor coating materials based on molecularly imprinted polymers (MIPs). The concept of using enzyme-like, synthetic MIPs as substance specific receptors is well known from mainly bio and life science applications detecting liquid analytes [1, 2]. The objective of our study is to use MIPs for detecting explosives vapours. These coating materials can be used in combination with different sensor concepts, e.g. mass specific sensors (QCM, SAW) or field effect transducers (FET).

\section{Principle of Molecular Imprinting}

The technique of molecular imprinting allows the formation of specific recognition sites in macromolecules. In this process, functional and cross-linking monomers are copolymerised in the presence of a target analyte (template). The functional monomers form a complex with the imprint molecule and in the subsequent polymerisation the functional groups are held in position by the highly cross-linked structure. Subsequent removal of the template reveals binding sites that are complementary in size and shape to the analyte (see Fig. 1). The complex between monomers and template can be formed via reversible covalent bonds [1] or via non-covalent interactions $[2,3,4]$ such as hydrogen bonds. For TNT or DNT as template it is not possible to use the covalent approach. Furthermore with the non-covalent approach we are able to use a large pool of functional monomers that are commonly used in the field of molecular imprinting. Because of the known problem that nitroaromatics are weak hydrogen bond acceptors [5], we used for the synthesis of the MIPs several acrylates with different functional groups as monomers (e.g. acrylamide, acrylic acid) and ethylene glycol dimethacrylate (EGDMA) as cross linking agent. 


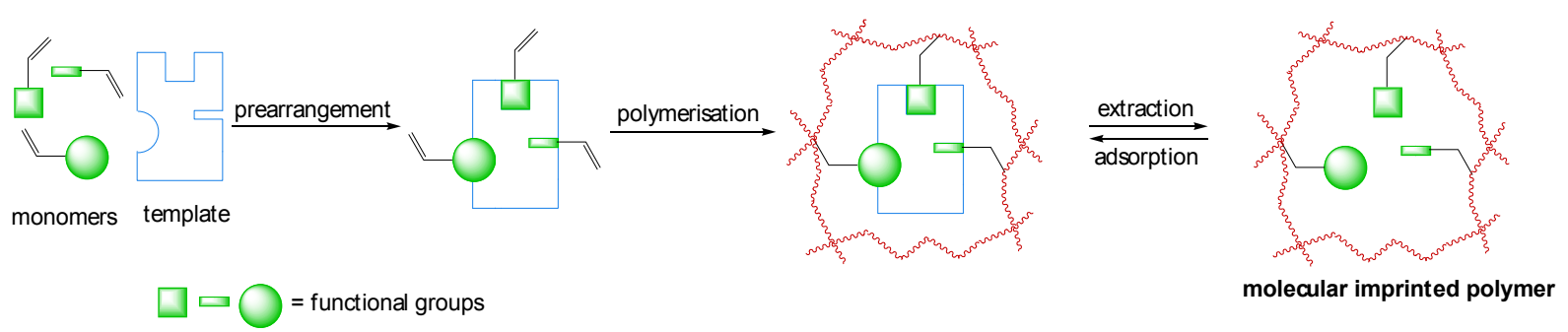

Fig. 1. Principle of molecular imprinting.

\section{Synthesis}

Direct synthesis of the imprinted and nonimprinted layers as thin film coatings on so called quartz crystal micro balances (QCMs) was performed in first experiments via manual spray-coating with an art air-brush gun. In later experiments we used a nanoplotter(GeSiM, Grosserkmannsdorf) for applying the MIP solution onto the sensor surface followed by UV polymerisation (UV starter Irgacure type) with a point lamp (Dymax). To adjust the layer thickness to the requirements of different sensor technologies we also used a spin coater to decrease the layer thickness down to some nanometers. As porogenic solvents acetonitrile (ACN), chloroform $\left(\mathrm{CHCl}_{3}\right)$ or dimethylformamide (DMF) were tested. To verify the enhanced uptake of TNT (or other substances) from the vapour stream, the imprinted polymer type was always tested against the nonimprinted correspondent using a special TNT gas generator [6]. The TNT uptake was directly measured via the decreasing QCM frequency of the treated MIP layers. As empirically found by Sauerbrey [7] the frequency of a QCM decreases linearly up to about 2 per cent of the quartz mass as a mass attachment occurs. For QCM measurements a commercial gaslab (ifak, Magdeburg) with quartzes having a ground frequency of $10 \mathrm{MHz}$ were used.
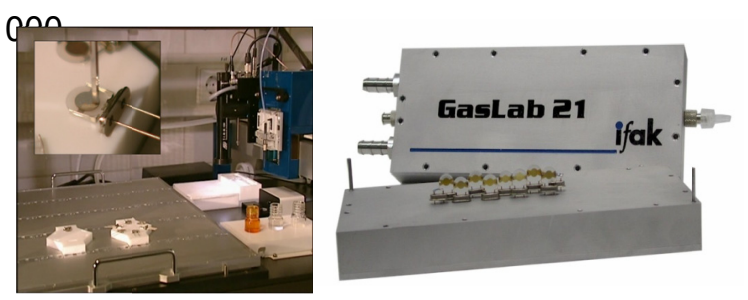

Fig. 2. Coating of QCMs via nanoplotter (Fa.GeSiM), QCM test modul (ifak).

Depending on the actual sensor type, its surface material (e.g. silver, gold, quartz etc.), its roughness and its geometric dimensions, a suitable and reproducible coating procedure must be worked out. While we started testing sufficient coating procedures using $10 \mathrm{MHz}$
QCMs with rough silver or gold electrodes we got some adhesion problems when we proceeded to coat polished gold electrodes. Especially, the necessary washing procedure of the polymerised MIP coated QCMs using organic solvents then sometimes lead to a partly peeling off of the produced MIP layers. Therefore, we looked out for a suitable pretreatment procedure to enhance the adhesion on polished surfaces. We tested different thiol anchor groups and other methods such as "grafting from" or RAFT-polymerisation to achieve an immobilisation of the afterwards polymerised MIP material. The best results were achieved using $24 \mathrm{~h}$ pretreatment of the QCM gold electrodes with pentene-1 thiol which was synthesized in a one-step reaction using allylmagnesium bromide and ethylene thiol. The thiol anchor was covalently bound to the polished gold electrode over nearly the whole surface.

\section{Results and discussion}

Performance tests of nanoplotted TNT imprinted and non-imprinted PMAA coated QCMs showed that we achieved an imprint effect with this coating technology. The MIP performance strongly depends on the used monomer and solvent. For example results obtained with manual spray-coating showed that polyacrylamide in combination with $\mathrm{CHCl}_{3}$ showed the best adsorption of TNT per hour (150 pg per mg MIP per hour) while DMF and ACN showed minor adsorption tendency for TNT. The second best sensitivity until now was achieved for MAA also synthesized in $\mathrm{CHCl}_{3}$. As could be seen in Figure 3, the adsorption of TNT is reversible if a pure nitrogen gas flow is used instead of the TNT vapour generator. Similar results were achieved for 2,4-DNT as template. Preliminary measurements with respect to the potential cross-sensitivity of TNTimprinted MIPs using 2,4-DNT as test gas provided no measurable adsorption amounts as well as DNT-imprinted MIPs did not show measurable affinity for TNT. 


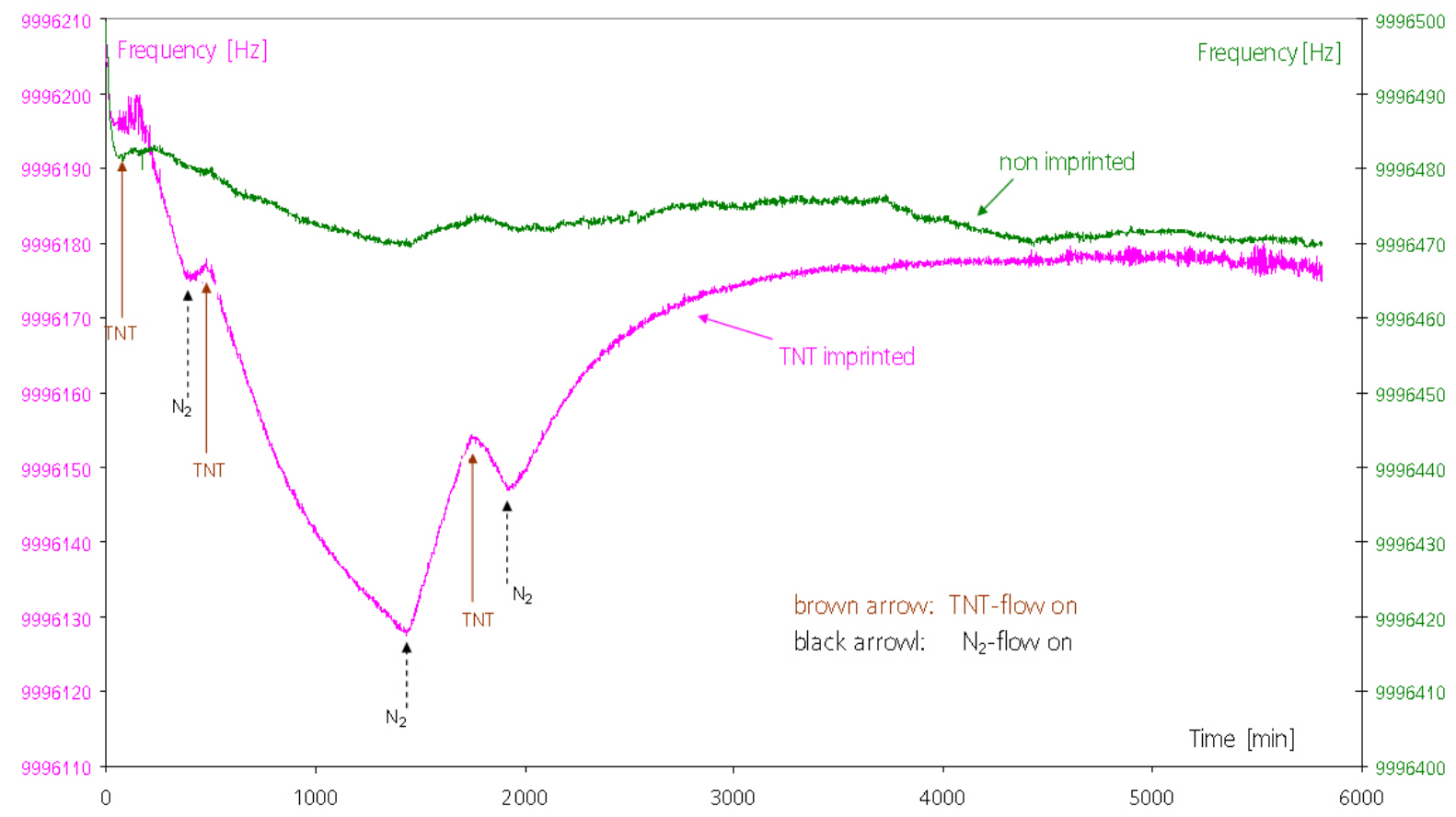

Fig.3. Adsorption and desorption of gaseous TNT exemplarily for a non-imprinted (right axis) and a TNT imprinted PMAA layered QCM (left axis).

\section{References}

[1] G. Wulff, A. Sarhan, Über die Anwendung von enzymanalog gebauten Polymeren zur Racemattrennung, Angew. Chem. 84, 364 (1972); doi: 10.1002/ange.19720840838

[2] B. Sellergren, Molecularly Imprinted Polymers Man-MadeMimics of Antibodies and theirApplications in AnalyticalChemistry, Elsevier Science B.V., Amsterdam, 2001

[3] M. Komiyama, T. Takeuchi, T. Mukawa, H. Asanuma, Molecular Imprinting, Wiley- $\mathrm{VCH}$, 2003, ISBN: 3-527-30569-6

[4] K. Mosbach, K. Haupt, Some new developments and challenges in non-covalent molecular imprinting technology, J. of Molecular Recognition 11, 62-68 (1998), doi: 10.1002/(SICI)1099-1352(199812)11:1/6 $<62:$ :AID-JMR391>3.0.CO;2-5

[5] W. F. Baitinger, P. R. Schleyer, T. Murty, L. Robinson, Nitro groups as proton acceptors in hydrogen bonding, Tetrahedron 20,1635-1637 (1964), doi: 10.1016/S0040-4020(01)99161-6

[6] G. Bunte, J. Hürttlen, G. Unkelbach, K. Niedergall, $H$. Krause, Imprinted polymers for gas phase detection of TNT, MIP2004 - Third International Workshop on Molecularly Imprinted Polymers, Cardiff, UK, September 12-15th, 2004

[7] G. Sauerbrey, Verwendung von Schwingquarzen zur Wägung dünner Schichten und zur Mikrowägung, Zeitschrift für Physik 155, 206222, (1959), doi: 10.1007/BF0133793 